\title{
First results from a laboratory hypertelescope using single-mode fibers
}

\author{
F. Patru ${ }^{1}$, D. Mourard ${ }^{1}$, J.-M. Clausse ${ }^{1}$, L. Delage ${ }^{2}$, F. Reynaud ${ }^{2}$, M. Dubreuil ${ }^{1}$, D. Bonneau ${ }^{1}$, S. Bosio ${ }^{1}$, Y. Bresson ${ }^{1}$, \\ Y. Hugues ${ }^{1}$, O. Lardière ${ }^{3}$, and A. Roussel ${ }^{1}$
}

1 Observatoire de la Côte d'Azur, Dpt. Gemini, UMR CNRS 6203, avenue Copernic, 06130 Grasse, France e-mail: Denis . Mourard@obs-azur . fr

2 XLIM, Dpt. Photonique, UMR CNRS 6172, 123 avenue Albert Thomas, 87060 Limoges Cedex, France

3 Adaptive Optics Lab, Engineering Lab Wing B133, University of Victoria, PO Box 3055 STN CSC, Victoria, BC, V8W 3P6, Canada

Received 29 September 2007 / Accepted 19 October 2007

\section{ABSTRACT}

\begin{abstract}
Context. In the future, giant optical interferometric arrays will be developed with kilometric baselines and a large number of telescopes. Such arrays could have direct imaging capabilities if optimized beam combiners are used.

Aims. This paper aims at studying the performance of an interferometric beam combiner using single mode fibers and in the frame of a hypertelescope.

Methods. A laboratory testbed called SIRIUS was developed. We describe the general concept, the technical specifications and the results obtained. These results are analyzed with the help of a numerical simulator.

Results. Direct images were obtained at the densified focus of SIRIUS. We show that the fibers greatly ease the pupil rearrangement. They also greatly improve the quality and the stability of the direct image. The computed images allow us to reproduce the effects of differential photometry and the influence of optical path difference variations. Optical path difference errors less than $\lambda / 10$ and differential photometries less than $60 \%$ are required to keep the quality of the direct image.

Conclusions. These results demonstrate the great potential of direct imaging interferometric beam combiners for future optical large arrays. The excellent comparison between experience and simulation clearly shows the simplicity of the fibered pupil densifier. It also gives us a great confidence in the extrapolation of these results and specifications for future arrays with a very large number of apertures.
\end{abstract}

Key words. instrumentation: high angular resolution - instrumentation: interferometers - telescopes - methods: observational

\section{Introduction}

Conceptual design studies are mandatory to define future kilometric optical interferometers. In this context, an interferometric testbed, called SIRIUS, has been developed to study the recombination schemes for direct imaging with the densified pupil concept (Labeyrie 1996). Pupil densification is basically a remapping of a dilute array of input apertures onto an intermediate plane that is largely filled, thus permitting direct imaging of the source. It has been shown that, compared to the Fizeau mode (Fizeau 1868), the pupil densification improves the sensitivity gain without loss of field of view (Lardière et al. 2007).

SIRIUS aims at studying the performance of densified pupil beam combination schemes, for the long term purpose of direct imaging of astronomical complex sources. A simulation software has been written for helping in the definition of the testbed and for correctly understanding the results. This simulator, called HYPERTEL, consists of two main parts. The first one aims at simulating images of a complex source as seen through an interferometer and for different types of beam combination. The second part of the program analyzes the simulated images or the SIRIUS raw images. It aims at measuring the characteristics of the point spread function (PSF) or, for a classical aperture synthesis analysis, the visibilities.
The main purpose of SIRIUS is to allow identification of the technical requirements like differential photometry and cophasing tolerances. The technical specifications are derived from criteria measuring the quality of the direct image in the focal plane, in terms of intensity distribution. The degradations are quantified by studying the on-axis irradiance decrease of the central peak or the energy dilution from the central peak to the surrounding halo.

Four main criteria are used here to characterize the PSF (Patru 2007; Patru et al. 2008). The on-axis intensity $I_{0}$ is equal to the amplitude of the central peak. The encircled energy $E_{0} / E_{\text {tot }}$ is defined as the ratio of the fraction of energy contained in the central peak from the total energy in the image. The maximal halo level $I_{1} / I_{0}$ is defined as the ratio of the intensity of the maximal side-lobe and the intensity of the central peak. The full-width half-maximum $(F W H M)$ of the central peak is also considered.

In a first step, a direct Fizeau scheme was achieved (Patru et al. 2004) that reproduces the VLTI interferometer (4 UT) with a pupil mask on a collimated beam of diameter $D_{\text {pup }}=70 \mathrm{~mm}$. The direct Fizeau images were analyzed with the HYPERTEL software (Patru et al. 2008) in the aperture synthesis mode but great instrumental difficulties were encountered. First, the direct Fizeau scheme does not allow the position of 


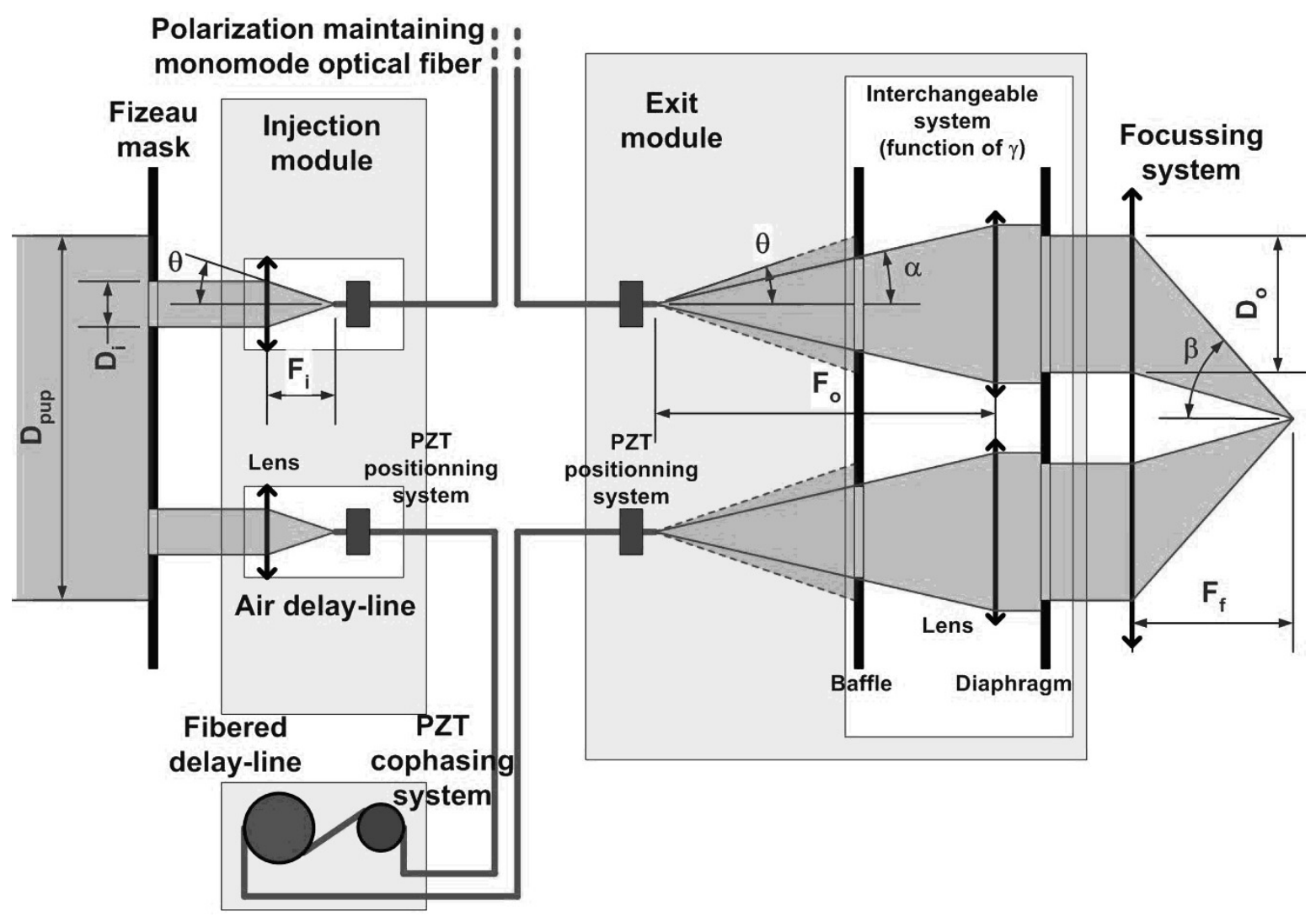

Fig. 1. Principle of the fibered pupil densifier SIRIUS, in a simplified version with only two pupils. The pupils are rearranged by preserving the input/output layout of the array and by increasing the diameters of the sub-pupils in the output subsystem. Each beam of the entrance pupil mask is injected in a polarization maintaining single mode fiber, through an injection module. An air delay-line, integrated in the injection module, is used on each arm to correct the intrinsic optical path defaults of the system. The lengths of the fibers are adjusted to cancel the differential chromatic dispersion between the fibers. Fiber stretching principle is used for this parameter of the system. The exit module of the fibers collimates each truncated Gaussian beam with the right diameter of the diaphragm. Three interchangeable sets of collimation allow three levels of densification: Fizeau, partial densification and maximal densification.

each beam to be individually adjusted at the common focus. Consequently the superposition was very poor. Secondly, the internal optical path differences were not completely negligible. The superposition and the cophasing of the system were very dependent of the external conditions (temperature, effective alignment...) and no quantitative analysis was really obtained. This first step has mainly allowed us to fix all the problems in terms of light contamination, thermal and mechanical stability, and also in terms of automated operations for avoiding the influence of human interventions.

The pupil densification scheme was then developed with the goal of using up to 8 single-mode fibers (Reynaud et al. 2001; Delage et al. 2000). Single-mode fibers allow spatial filtering and offer a more compact and flexible solution for beam combination (Patru et al. 2007). Furthermore, using single-mode fibers allows a simple optical implementation of the hypertelescope principle. For this work, we used a mask reproducing the UT1UT2-UT3-UT4 configuration of the VLT Interferometer. Each UT is represented by a hole of $2.7 \mathrm{~mm}$ and the largest baseline UT1-UT4 has a length of about $47 \mathrm{~mm}$ on the mask.

Section 2 presents the general and detailed specifications of the fibered pupil densifier. The results and comparisons with numerical simulations are shown in Section 3. Section 4 discusses the consequences of these results for the specifications of future ambitious long baseline optical interferometers.

\section{Description of the optical system}

The concept of the fibered pupil densifier is schematically presented in Fig. 1. We use polarization maintaining singlemode optical fibers (Bow-tie HP600) in the visible wavelengths $(635 \mathrm{~nm})$. The densifier is divided into three main subsystems:

- the injection module, to inject the light of each beam into the fibers;

- the fiber arm, which is also used to control the propagation of the light;

- the exit module, to define the output densified pupil and to properly recombine the beams on the CCD detector at the densified focus.

\subsection{Injection module}

This is the first system encountered by the optical beams. The injection in single mode fibers is analyzed in terms of coupling efficiency (transmitted flux for the differential photometry analysis), polarization, and optical path difference control, in order to maintain coherence between the beams.

SIRIUS operates at a wavelength $\lambda=635 \mathrm{~nm}$. Our fibers have a central beam radius $\omega_{0}=1.64 \mu \mathrm{m}$. To properly inject 


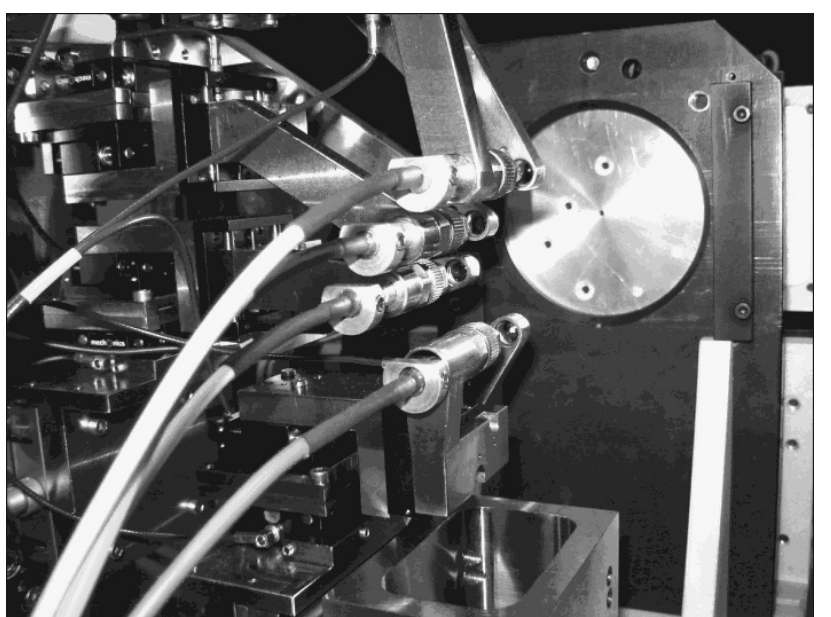

Fig. 2. Injection module of SIRIUS. Each beams from the 4 UTs configuration of the VLTI is injected into a fiber through an injection lens. Each fiber is supported by three piezo-electric translation stages to bring the fiber head at the focus of the lens.

the light into the fibers, the aperture angles $\theta$ of the input beams should satisfy the relation (Saleh Bahaa 1991):

$\theta=\frac{\omega(z)}{z}=\frac{\lambda}{\pi \omega_{0}}$

For a given input diameter $D_{\mathrm{i}}$, the input focus $F_{\mathrm{i}}$ of the injection lens follows:

$F_{\mathrm{i}}=\frac{D_{\mathrm{i}}}{2 \tan (\theta)}$.

In order to correctly fix the numbers on the mechanical requirements for the injection system, we developed an optical model of the injection of a beam in a single mode fiber. This simulation analyzes the flux variation as a function of transverse and longitudinal (defocus) displacements, and of tilts of the fiber at the focus. These calculations show that a loss of $10 \%$ of flux is produced by a shift of $0.4 \mu \mathrm{m}$ or by a defocus of $10 \mu \mathrm{m}$ of the input fiber core. A tilt of the fiber of $2^{\circ}$ induces a loss of $6 \%$ of the coupling rate. These tolerances are known as the "antenna diagram" and do not depend on the pupil configuration.

For each arm of the interferometer, the polarization axes of the fibers have to be precisely superimposed to avoid differential polarization effects. It has already been demonstrated (Koechlin 1981) that the influence of a differential orientation $\zeta$ of the polarization axis between two beams creates a visibility attenuation equal to $\cos (\zeta)$. A visibility loss of $10^{-3}$ corresponds to $\zeta=2.5^{\circ}$.

Mechanically speaking, the injection module is built around different elements. The first piece is an achromatic lens focusing the beam on the fiber core. The fiber itself is supported by a mechanical arm motorized by 3 piezo-electric translation stages (Fig. 2). These translation stages have an accuracy of $30 \mathrm{~nm}$, which is sufficient to maximize the flux coupled in the fibers and to equalize the differential photometry. They also have a very useful stroke of $\pm 4 \mathrm{~mm}$, allowing large scans for finding the beams and eventually easy reconfiguration of the pupil geometry. A tilt of the fibers below $1^{\circ}$ is ensured by construction. The slow axis of the polarization maintaining fibers are marked so as to align the polarization axis with an accuracy higher than $\zeta=2.5^{\circ}$, by turning the fiber head in its connector. The injection lens and the fiber with its mount are installed on a manual translation stage, allowing compensation of the optical path defaults

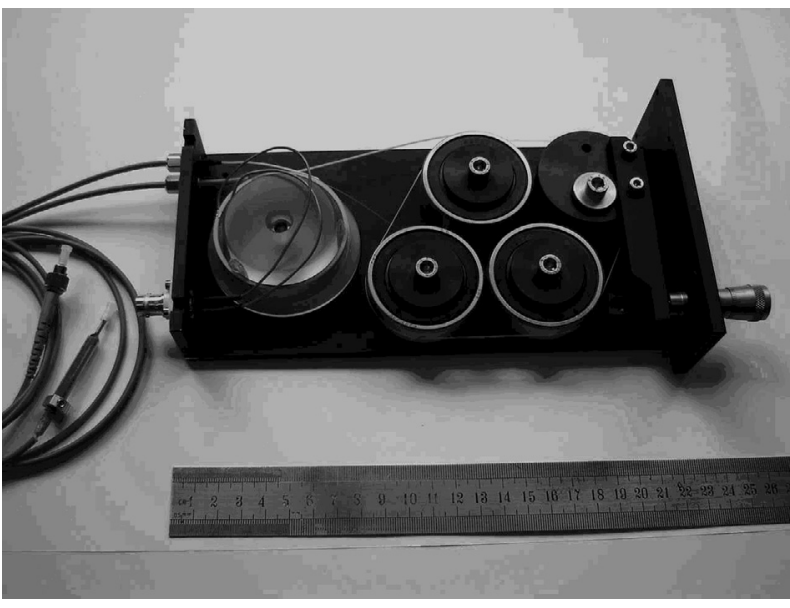

Fig. 3. The SIRIUS fibered delay-line. This system was developed by some of us (FR, LD) and is now purchased by the LEUKOS factory (www. leukos-systems. com). The fibered delay-line controls the chromatic dispersion by rolling and stretching the fibre around 4 rollers. The cophasing system is based on a fibre length control system, where the fibre is wound around a piezo-electric ceramic.

of the system. This air delay line has a stroke of $8 \mathrm{~mm}$ and a resolution of a few microns.

\subsection{Fibered delay-line}

The use of fibers may induce differential chromatic dispersion (Tango 1990) if their lengths are not correctly equalized. At visible wavelengths a difference of length less than $100 \mu \mathrm{m}$ is necessary for the type of fibers we use.

First, the geometrical lengths of the fibers $(10 \mathrm{~m}$ in our testbed) are equalized with an accuracy of $\pm 2 \mathrm{~mm}$ by polishing the tips of the fibers. Then, one fiber (ideally the longest one) is considered as the reference and the others are manually stretched to precisely balance their optical lengths, with respect to the reference one. For this, the channeled spectrum method (Shang 1981; Vergnole 2004) was applied on a specific testbed (Patru et al. 2006). The fibered delay-line can linearly stretch the fiber with a resolution better than $50 \mu \mathrm{m}$ and a stroke of about $6 \mathrm{~mm}$ in equivalent air path. The air delay-line of the injection module is then used to compensate the delay induced by the stretching of the fiber.

The raw coherencing of the combiner is done with the air delay lines, whereas the fine cophasing is ensured by a fibered cophasing system. For this purpose, the fiber is wound around a piezo-electric ceramic driven by a voltage generator. This fiber length control system allows variations of the optical path difference (OPD). The stroke of the cophasing module is limited to $100 \mu \mathrm{m}$ to avoid the introduction of undesirable chromatic dispersion. The resolution reaches $\lambda / 100$ with a servo control.

The manual and servo-driven stretching of the fiber are made in one single unit. One part of the fiber is rolled and manually stretched around 4 ball bearings, whereas the second part of the fiber is wound on a piezo-electric ceramic. The unit is very compact and their dimensions allow then to be put in a standard electronic rack that can be thermally controlled (Fig. 3).

\subsection{Output module}

The functions of the exit module are to remap the entrance pupil geometry, to zoom the individual sub pupils depending on the 


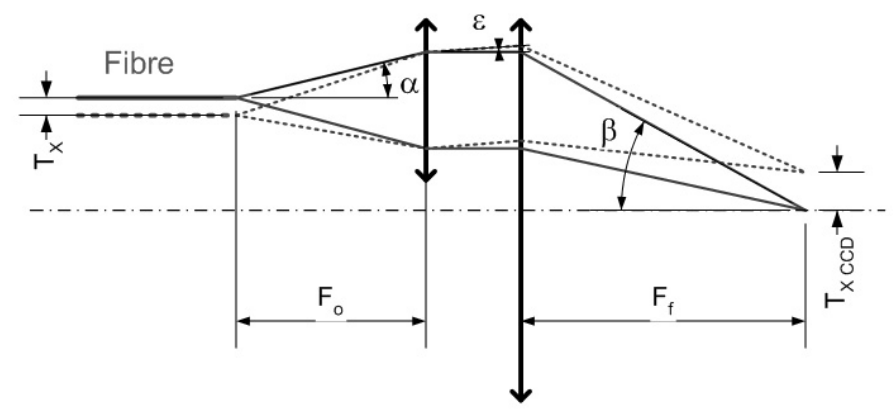

Fig. 4. Superposition of the beams at the imaging focus. The output beam of the fiber first encounters the individual collimating lens. Then all the collimated beams are focused at the densified focus by the general collimating lens. A radial shift $T_{X}$ CCD of the sub-image on the CCD is due to a radial shift $T_{X}$ or a defocus $T_{Z}$ of the fiber head.

densification factor, to collimate each beam and then to form the common image on the CCD detector.

The focal length $F_{\mathrm{o}}$ of the individual collimating lense (Fig. 4) is chosen to properly adjust the diaphragm diameter $D_{\text {o }}$ with the Gaussian intensity distribution at the output of each fiber. It has been shown (Patru et al. 2007) that the width of the diaphragms can be adjusted to optimize the imaging properties. The corresponding focal length $F_{\mathrm{o}}$ can be written as:

$F_{\mathrm{o}}=0.18 \frac{\lambda}{\omega_{0}} D_{\mathrm{o}}$

This condition keeps the same aperture angle of all the emerging beams $\left(\tan (\alpha)=D_{\mathrm{o}} / F_{\mathrm{o}}\right)$, whatever the input configuration and whatever the level of densification. In the case of a multi-axial recombination, the beams are properly focused on the imaging focus by the global collimating lens if they are rightly collimated and if they are parallel to the optical axis. To specify the requirements on the mechanical mounts of the fiber's output, we consider the influence on the individual images formed on the CCD detector. The image after the fiber output is a pseudo-truncated Gaussian spot with a full width at half maximum (FWHM) of $2.2 \omega_{0}$ (Patru et al. 2007). This spot is re-imaged on the CCD with a magnification $G_{\mathrm{o}}=F_{\mathrm{f}} / F_{\mathrm{o}}$, where $F_{\mathrm{f}}$ is the focal length of the focussing system. We have considered the on-axis intensity and the FWHM as the main criteria for the image quality of the individual beams. A simple calculation shows that a shift of $T_{\mathrm{X}}=0.32 \omega_{0}$ corresponds to a change of $10 \%$ on the on-axis irradiance. On the CCD, this corresponds to a radial shift $T_{X \mathrm{CCD}}$ :

$T_{X \mathrm{CCD}}=0.32 \omega_{0} G_{\mathrm{o}}$.

In the same way, a variation of $10 \%$ of the on-axis irradiance also corresponds to a defocus of $T_{Z \mathrm{CCD}}=T_{X \mathrm{CCD}} / \tan (\beta)$ or $T_{Z}=$ $T_{x} / \tan (\alpha)$. By using the relation $\tan (\beta)=D_{\text {pup }} / 2 F_{\mathrm{f}}$, where $\beta$ is the maximum aperture angle of the focalized beams on the CCD (Fig. 4), and the relation $\tan (\alpha)=\frac{D_{\mathrm{o}}}{2 F_{\mathrm{o}}}$, we can write:

$T_{Z \mathrm{CCD}}=\frac{2 F_{\mathrm{f}}}{D_{\text {pup }}} T_{X \mathrm{CCD}}, \quad T_{Z}=\frac{2 F_{\mathrm{o}}}{D_{\mathrm{o}}} T_{X}$.

In the case of the maximal densification mode, the magnification equals to $G=69$, with a typical focal length $F_{f}=4 \mathrm{~m}$ to provide a convenient sampling. The corresponding tolerances for positioning the CCD are then $T_{X \text { CCD }}=25 \mu \mathrm{m}$ and $T_{Z \text { CCD }}=3 \mathrm{~mm}$. The tolerances for positioning the output fiber head at the focus of the lens correspond to a transversal shift of $0.38 \mathrm{~mm}$ resulting in a defocus of $3.1 \mu \mathrm{m}$. We notice that a defocus is less critical

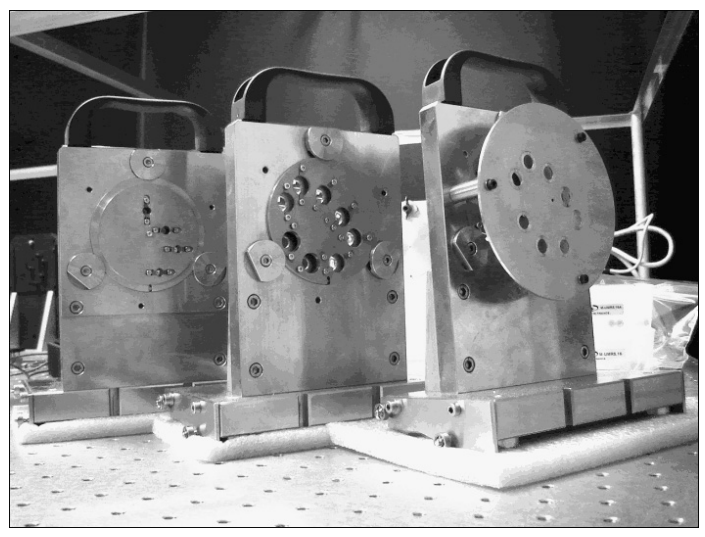

Fig. 5. The three interchangeable collimating and densifying systems of SIRIUS. On each, eight lenses are foreseen but only 4 of them were used for this work.

Table 1. Main parameters of the different configurations of SIRIUS: densification factor $\gamma$, diameter of the input sub-pupil $D_{\mathrm{i}}$, focal length of the injection lens $F_{\mathrm{i}}$, input aperture angle $\theta$, diameter of the output diaphragm $D_{\mathrm{o}}$, focal length of the collimation lens $F_{\mathrm{o}}$, and output aperture angle $\alpha$.

\begin{tabular}{lccc}
\hline \hline Densification & $\gamma_{\max }$ & $\gamma_{\max } / 2$ & Fizeau \\
\hline$\gamma$ & 5.5 & 2.5 & 1 \\
$D_{\mathrm{i}}(\mathrm{mm})$ & 2.7 & 2.7 & 2.7 \\
$F_{\mathrm{i}}(\mathrm{mm})$ & 11.7 & 11.7 & 11.7 \\
$\theta\left({ }^{\circ}\right)$ & 7 & 7 & 7 \\
$D_{\mathrm{o}}(\mathrm{mm})$ & 14.85 & 6.75 & 2.7 \\
$F_{\mathrm{o}}(\mathrm{mm})$ & 58.1 & 26.4 & 10.6 \\
$\alpha\left({ }^{\circ}\right)$ & 7 & 7 & 7 \\
\hline
\end{tabular}

than a radial shift. These tolerances remain the same whatever the entrance pupil configuration. We summarize in Table 1 the whole parameters of the SIRIUS fibered pupil densifier.

The mechanical design of the output module is very similar to the injection module but without the air delay lines. Each fiber head is positioned at the focus of a collimating lens with three piezo-electric translation stages. Collimating lenses in front of each of the fibers are mounted on a second module (Fig. 5). The polarization axes are aligned by fixing the slow axis in the direction of the FC-PC connector.

To superimpose accurately the sub-images at the imaging focus, the tilt and the focus of each output beam are adjusted. In practice, the sub-images are superimposed with an accuracy of a few percent of the half-width at half maximum of intensity. This limits the on-axis irradiance bias to below $1 \%$.

Three interchangeable systems are available to collimate the emerging beams with the three levels of densification (Table 1 and Fig. 5). In the maximum densification case, where the output sub-pupils are tangent, a baffle system adjusts the aperture of the beams to fit the sub-pupil size with the collimating lens and to avoid light crosstalk between the beams.

\subsection{Operating SIRIUS}

The differential polarization and the differential chromatic dispersion of the fibers were minimized on a separated testbed (Patru et al. 2006). The initial alignment consists of feeding the light into each fiber and bringing the emerging beams on the 

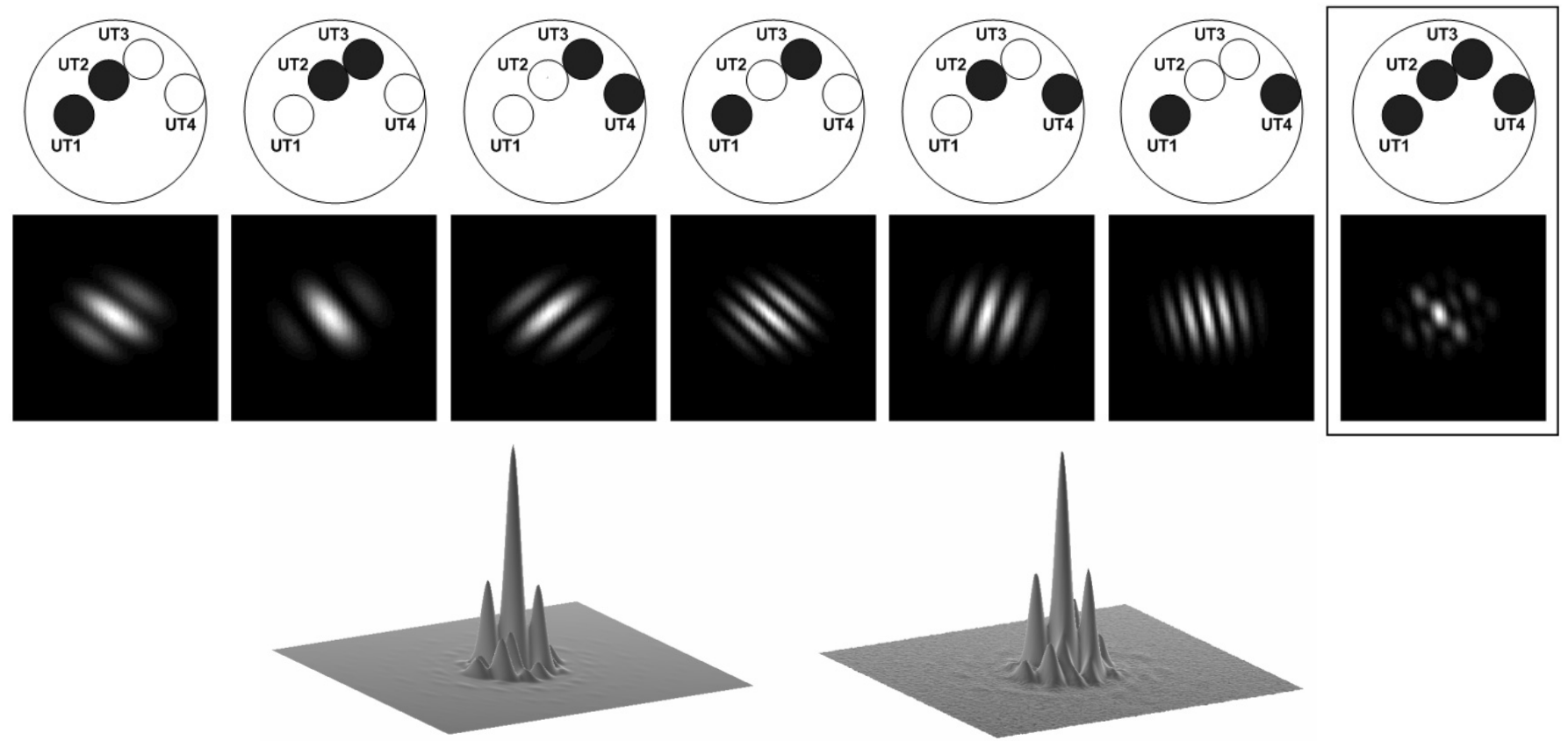

Fig. 6. Densified images (top) obtained of SIRIUS with 2 UTs (baseline by baseline) and 4 UTs (superposition of all the cophased beams). The comparison between the simulated PSF (bottom left) and the experimental PSF (bottom right) shows a very encouraging agreement.

CCD detector. Then, the following procedure is used as described step by step:

- maximization of the injected flux in the fibers, by adjusting the piezo-electric translation stages of the injection module;

- equalization of the photometric signals, by defocusing the brightest beams at the injection;

- focussing of the emerging beams with the $Z$ piezo-electric translation stages of the exit module, so as to minimize the FWHM of each sub-image;

- collimation of the emerging beams by moving the $X$ and $Y$ piezo-electric translation stages on the exit module in order to superimpose properly the sub-images on the CCD detector;

- coherencing of the beams by pairs (Fig. 6), by manually adjusting the air delay-lines;

- cophasing of the beams by pairs, by driving the piezoelectric cophasing systems.

All these operations are achieved with the control of the images on the CCD detector. A real time processing of the images allows the measurements of the integrated flux, the FWHM and the photometric barycenter of the image. An automatic system was developed to avoid vibrations or perturbations due to the operator during alignment and measurement phases. An external supervisor communicates with each sub-system: the CCD detector, the entrance shutter of the light source, the calibration mask (to select one beam, two beams, or all the beams), the piezo-electric translation stages of the injection and exit modules, and the piezo-electric cophasing systems. This supervisor works with scripts containing the elementary commands. This is very useful for doing repetitive test sequences without user intervention. It also represents a good model for testing the automatic alignment and control of such a device.

\section{SIRIUS: experimental results}

\subsection{Characterization of the instrumental PSF}

Figure 6 shows the densified images obtained for each pair of telescopes and the densified images with 4 telescopes. There is
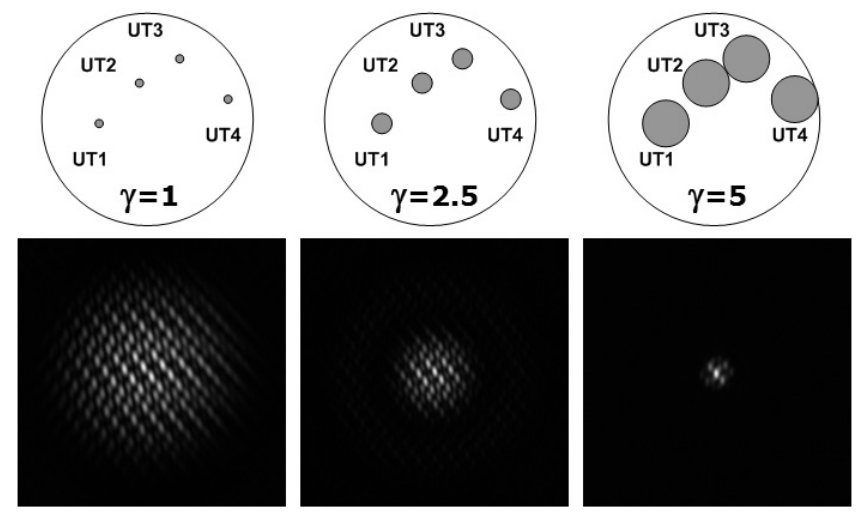

Fig. 7. Variable densification. Output pupil (top) and PSF (bottom) obtained on SIRIUS with the 4 UTs configuration in Fizeau mode (without fiber), in partial densification mode and in maximal densification mode (from left to right, respectively). The three images have the same maximum intensity level but the exposure times are completely different, clearly showing the intensification of the signal kept by the densification operation. For the Fizeau images, the exposure time is $250 \mathrm{~ms}$, whereas it is $25 \mathrm{~ms}$ in the partial densified image and $5 \mathrm{~ms}$ in the maximum densification image.

a very good fit between the simulations and the SIRIUS experiment, as shown at the bottom of the figure.

As foreseen in the simulation, the intensification of the signal with the increase of the densification factor can easily be seen on the densified images. For the same maximum amplitude in the image (Fig. 7), the exposure time in the maximal densification case is $5 \mathrm{~ms}$, versus $25 \mathrm{~ms}$ in the partial densification case. It corresponds to an intensification by a factor of 5 , which is in very good adequation with the densification factor difference $\left(\gamma_{\max } / \gamma_{\max / 2}\right)^{2}=(5.5 / 2.5)^{2}=4.9$. The exposure time in the Fizeau mode is about $250 \mathrm{~ms}$, but it is difficult to compare it with the densification mode, due to the fact that it was recorded without fibers and with another light source. 

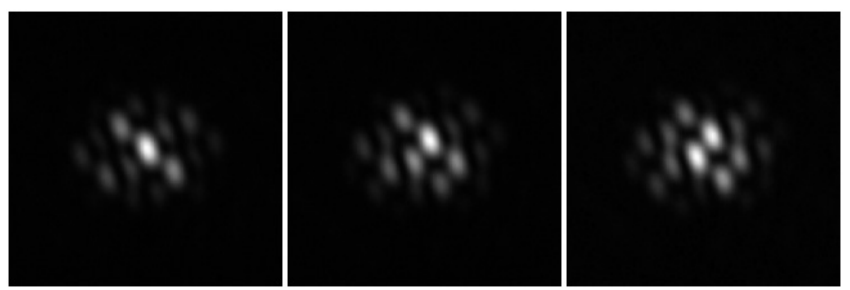

Fig. 8. Influence of the differential pistons on the PSF quality. An optical path is generated on the UT1 beam: $\Delta_{\mathrm{OPD}}=0, \Delta_{\mathrm{OPD}}=\lambda / 4, \Delta_{\mathrm{OPD}}=\lambda / 2$ (from left to right).

In the maximum densification case, the encircled energy in the central peak reaches $E_{0}=36 \%$, to be compared to $E_{0}=$ $38 \%$ with the simulations. In the partial densification case, the encircled energy reaches $E_{0}=4 \%$, to be compared to $E_{0}=6 \%$ in the theoretical case.

As the arrangement of the sub-pupils remains the same, the physical size of an element of resolution in the image remains the same whatever the densification factor. Therefore, the same spatial sampling is kept in the Fizeau and in the densification mode. The densified direct imaging field (DIF) is $\gamma$ times smaller than the Fizeau DIF (Fig. 7). The number of useful pixels is $\gamma^{2}$ times lower, whereas the intensity by pixels is $\gamma^{2}$ times higher. Thus, the densification reduces the information support to its optimal size (Lardière et al. 2007). This is another interesting point regarding the densification, particulary for the very long baselines, for which the interferometric fringes in the Fizeau image are very much spread.

The stability of the system was tested. We did not implement at this level the real time measurement of the pistons and the real time correction on the cophasing devices. We can observe slow fluctuations of the OPD, which are mainly due to the residual turbulence in the laboratory and to the thermal fluctuations. Experimentally, we observed that the residual turbulence decreased after around $30 \mathrm{~min}$. Temperature fluctuations of about $3{ }^{\circ} \mathrm{C}$ were observed over a day. In this passive operating mode of SIRIUS, the stability of the OPD is better than $\lambda / 10$ over $2 \mathrm{~min}$ and better than $\lambda / 4$ over $15 \mathrm{~min}$.

With the choice of spatial filtering with single mode fibers at the entrance of the densified combiner, the perturbations of the combined beams at the densified focus are only changes in photometry and variations of pistons. Thus, we used the SIRIUS testbed to deeply characterize these effects. The measurements were compared with the numerical simulations and are presented in the next subsections.

\subsection{Effects of the differential pistons on the PSF}

To quantitatively characterize the influence of the residual pistons on the image quality of the densified images, we introduced known delays and recorded the images over the time of stability of the experiment. Figures 8 and 10 show the evolution of the imaging parameters of the PSF with 4UTs as a function of the increasing piston on one of the four beams. For this purpose, the piston is increased step by step on the UT1 beam, from 0 to $\lambda / 2$. These measurements are achieved in less than $2 \mathrm{~min}$, so that the drifts due to the instability of the system are negligible.

The on-axis irradiance and the encircled energy decrease by $10 \%$ for an OPD of $\lambda / 4$. The halo level and the FWHM of the central peak are not greatly affected. For an OPD higher than $\lambda / 4$, it is more critical: first the halo increases and then the central peak is destroyed. As the OPD equals $\lambda / 2$, two peaks
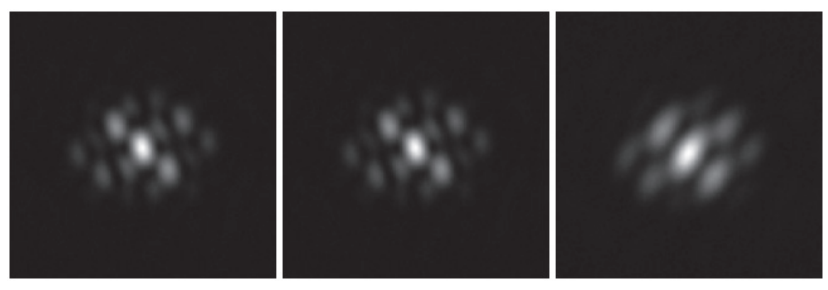

Fig. 9. Influence of the differential photometry on the PSF quality. The photometry is reduced on the UT1 beam: $\Delta_{\text {photom }}=0, \Delta_{\text {photom }}=50 \%$, $\Delta_{\text {photom }}=90 \%$ (from left to right).

predominate instead of one, so that the right peak of the image cannot be located.

We note a large discrepancy between simulations and experience, with respect to the halo level. The simulations do not take into account the truncation of the Gaussian beams. The diffraction envelope was approximated by a simple Gaussian, while in practice it is a Gaussian convolved by a Bessel (Patru et al. 2007). This envelope is the cause of a decrease of the contrast, with a central peak fainter than the side-lobes.

It has been shown that the cophasing is required to be $\lambda / 4$ to maintain the energy dilution from the central peak to the halo below $10 \%$. But this study is limited to an optical path variation of only one beam. In practice, phase fluctuations all over the beams have to be taken into account. A cophasing requirement of $\lambda / 10$ is chosen, which retains more than $90 \%$ of the energy in the central peak.

\subsection{Effects of the differential photometry on the PSF}

The next objective is to characterize the influence of the differential photometry on the PSF quality. Figures 9 and 11 show the evolution of the imaging parameters of the PSF with 4UTs as a function of the photometric decrease on one of the four beams. For this, the focus of the injection is modified on the UT1 beam, whereas the generated OPD is corrected with the cophasing system. The same operation is reproduced step by step in order to decrease the flux from 100 to $10 \%$.

The on-axis irradiance decreases, due to the photometric attenuation on the UT1 beam. However, the encircled energy of the central peak and the halo level are not greatly affected. Thus, the differential photometry induces a global photometric decrease in the image without destroying the quality of the direct image.

The FWHM of the central peak remains almost constant up to $60 \%$ of photometric loss. Thus, a differential photometry lower than $60 \%$ has little influence on the PSF quality, but involves essentially a global flux loss. Note that losing a beam is critical if few apertures are present. With a higher number of sub-pupils, the loss of one beam should have little influence.

\section{Discussion}

The first results obtained on SIRIUS show a very good agreement between simulated and experimental images. These results validate the simulation and the concept of the fibered pupil densifier.

The flexibility of the fibers is obviously very useful for rearranging the densified output pupil, depending on the deformation of the entrance pupil as seen from the sky. Moreover, this fibered system is very stable in time since the alignment is preserved during several months so as to keep the beams on the CCD detector. 

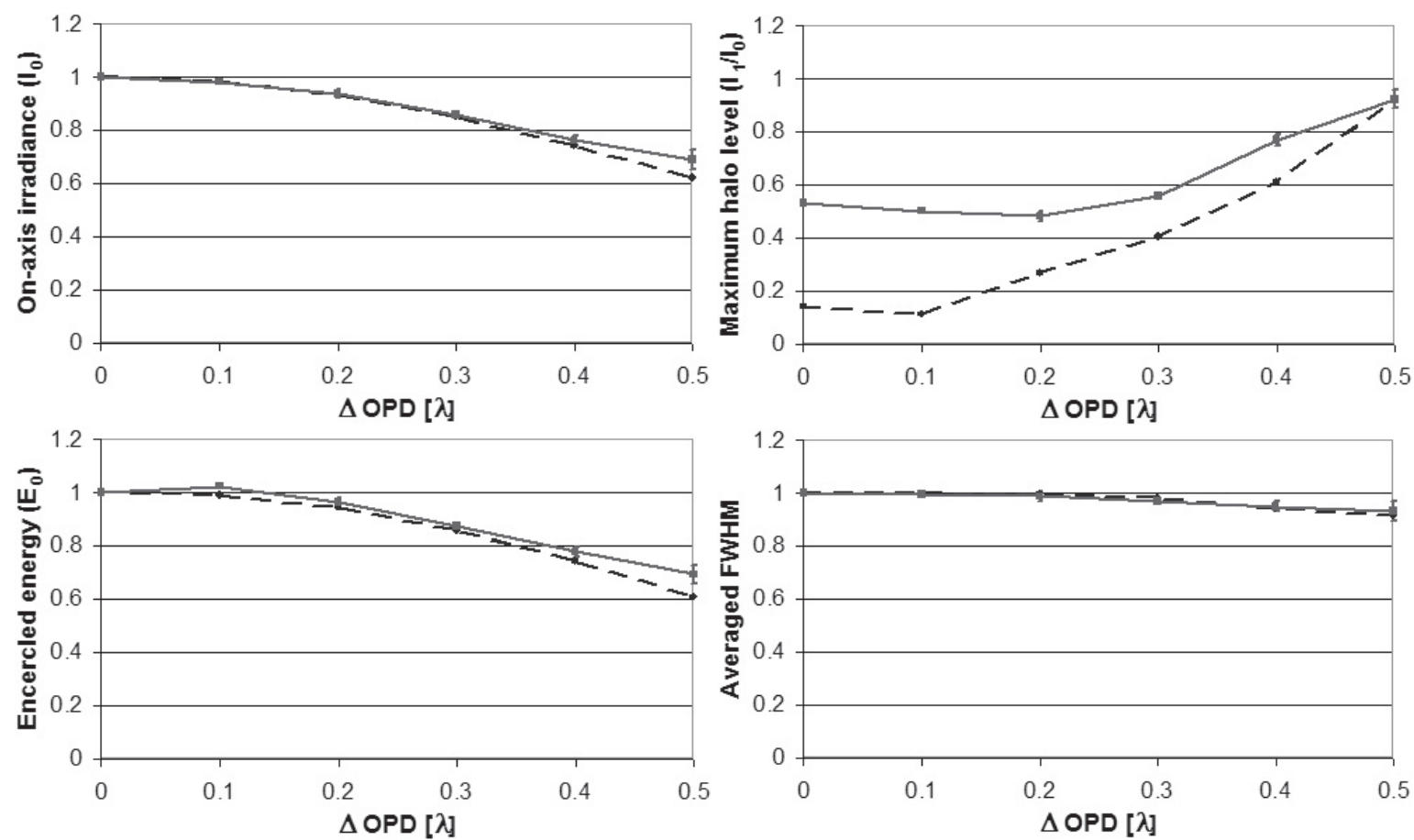

Fig. 10. Evolution of the imaging parameters of the PSF as a function of the piston on the UT1 beam. SIRIUS data (full line) and simulation data (dashed line).
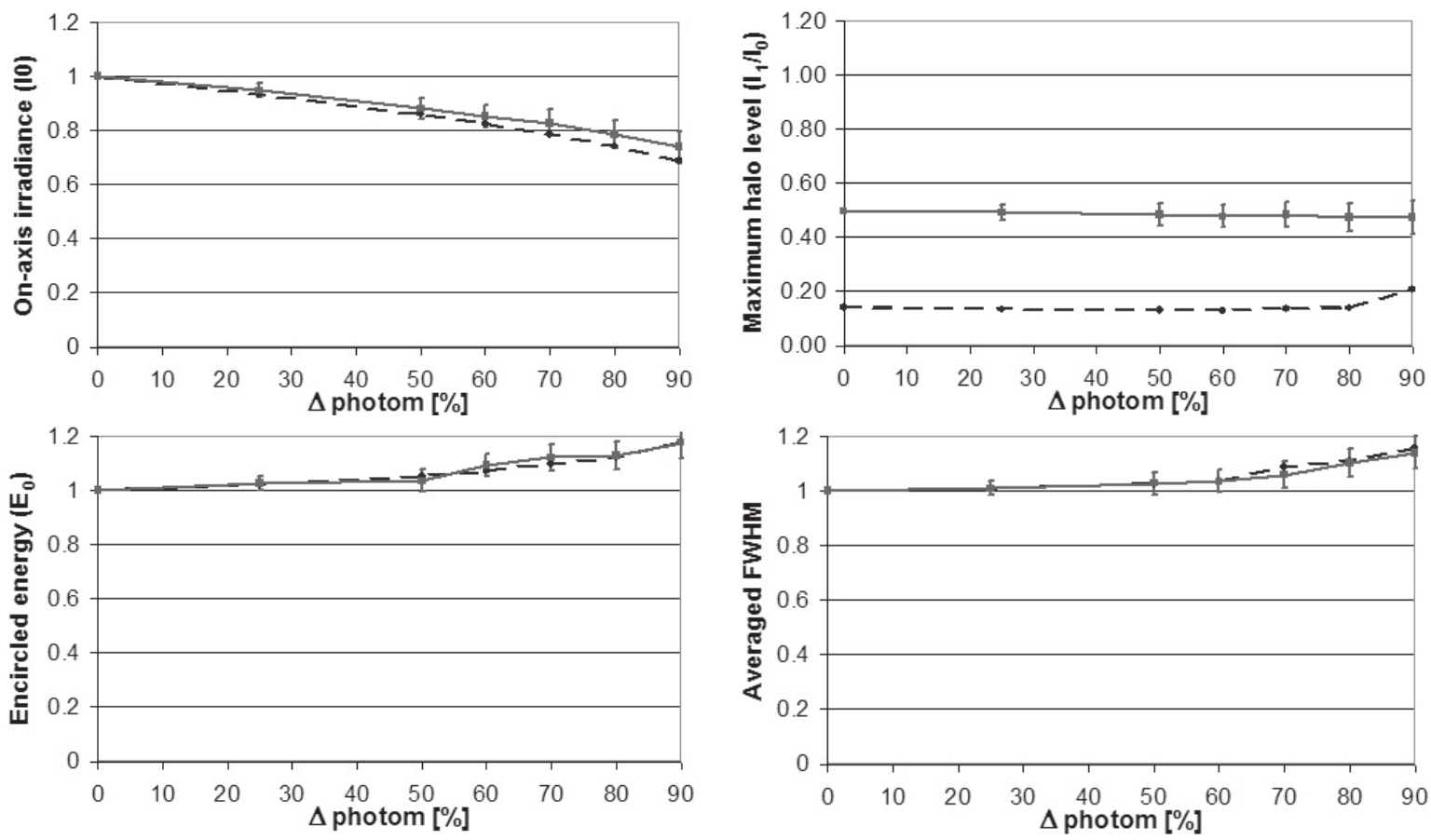

Fig. 11. Evolution of the imaging parameters of the PSF as a function of the photometry of the UT1 beam. SIRIUS data (full line) and simulation data (dashed line).

Another advantage of the fibers is to greatly ease the system adjustment. The interest is to decouple the entry and the exit of the assembly, which allow the separation of the problems. The alignment procedure concentrates on the degrees of freedom on the injection module, on the exit module, and then on the OPD equalization with the cophasing system. The densified image is properly restored if the beams remain in phase, at least at $\lambda / 10$.
Thanks to the spatial filtering properties of the single-mode fibers, any entrance perturbations are converted into photometric fluctuations and/or piston variations. We show that a densified image is much less sensitive to the differential photometry than to the differential pistons. A differential photometry of about $60 \%$ is tolerable. Thanks to the single-mode fibers, a disturbed wavefront is filtered at the entry by reducing the flux and 
by generating differential photometry. This does not greatly affect the quality of the direct image, but induces mainly a global decrease of the flux and a low uniform decrease of the contrast in the image. Thus, the spatial filtered image gains in quality and in stability.

However, the fibered recombiner is less sensitive than a classical recombiner. The injection in the fibers induces $20 \%$ of flux loss (Shaklan 1988), and the truncation of the Gaussian beams induces $8 \%$ of flux loss (Patru et al. 2007). This means that the intensification of the central peak is $35 \%$ fainter than a classical recombiner. The loss at the injection will increase in presence of atmospheric turbulence.

The deconvolution is greatly enhanced with fibers, when compared to a classical densifier. First, the diffraction envelope does not move, whatever the off-axis position of the observed point. Then, the form of the envelope is fixed and is imposed by the fiber and the truncation of the emerging beam. The beams are well superimposed because they are decorrelated from the turbulence upstream, so that the envelope is not affected by the differential tilt effects.

\section{Conclusion and prospects for the future}

The SIRIUS concept has a real potential for imaging compact objects on the sky. The fibered solution perfectly suits the ground interferometers with a simple optical scheme, which is easy to adjust. The main advantages are the decoupling between injection and output, the great ability of reconfiguration and the already demonstrated quality of calibration. The very nice agreement between the simulation and the experiment provides great confidence in the extrapolation of this principle for future long baseline arrays. In the middle term, applications are envisaged on the sky, to begin with pupil masking on a monolithic telescope, followed by a similar concept on an existing interferometer. The current arrays, the VLTI, CHARA, NPOI, MROI or OHANA, are able to produce direct images, by using new deconvolution techniques (Abe et al. 2004).

However, in presence of turbulence, the most significant cause of image degradation is the residual piston phases.
The active cophasing on the sky remains the most significant hard point. The next step is to study the admissible level of atmospheric turbulence and to deduce the required specifications for the injection in the fibers and the active cophasing of the beams to preserve an image of quality. Afterwards, it is planned that a fringe sensor unit will be designed and tested (Pedretti et al. 1999; Borkowski et al. 2004; Cassaing et al. 2006).

The final purpose of all these studies is to compare the imaging performances and applications between direct imaging and aperture synthesis imaging. The last step will be the addition of an integral-field spectrometer or a coronograph at the densified focus (Labeyrie 2003; Lardière et al. 2004a).

\section{References}

Abe, L., Aristidi, E., Vakili, F., Domiciano, A., \& Lopez, B. 2004, EAS Publ. Ser., 12, 275

Borkowski, V., Labeyrie, A., et al. 2004, A\&A, 429, 747

Cassaing, F., Sorrente, B., Mugnier, L., et al. 2006, Proc. SPIE, 6268, 104

Delage, L., Reynaud, F., \& Lannes, A. 2000, Appl. Opt., 39, 6406

Fizeau, A.H. 1868, C.R. Acad. Sci. Paris, 66, 932

Koechlin, L. 1981, Ph.D. Thesis, Univ. Nice

Labeyrie, A. 1996, A\&AS, 118, 517

Labeyrie, A., Le Coroller, H., Dejonghe, J., et al. 2003, Proc. SPIE, 4852, 236

Lardière, O., et al. 2004, EAS Publ. Ser., 12, 299

Lardière, O., Mourard, D., Patru, F., \& Carbillet, M. 2004, Proc. SPIE, 5491, 415

Lardière, O., et al. 2005, ESO VLTI Workshop, Garching

Lardière, O., Martinache, F., \& Patru, F. 2007, MNRAS, 375, 977

Patru, F. 2007, Ph.D. Thesis, Univ. de Nice Sophia-Antipolis

Patru, F., Mourard, D., Lardiere, O., et al. 2004, Proc. SPIE, 5491, 1613

Patru, F., Mourard, D., Delage, L., et al. 2006, Proc. SPIE, 6268, 57

Patru, F., Mourard, D., Lardière, O., \& lagarde, S., et al. 2007, MNRAS, 376, 1047

Patru F., Mourard., D., et al. 2008, MNRAS, in preparation

Pedretti E., Labeyrie A. 1999, A\&AS, 137, 543

Reynaud, F., Moser, R., Schindler, H., Blaas, D., \& Hinterdorfer, P. 2001, C.R. Acad. Sci. Paris, 2, 99

Saleh, Bahaa, E.A. 1991, (New York: John Wiley)

Shang, H.T. 1981, JOSA, 71, 1587

Shaklan, S., \& Roddier, F. 1988, Appl. Opt., 27, 2334

Tallon, M., \& Tallon-Bosc, I. 1992, A\&A, 253, 641

Tango, W.J. 1990, Appl. Opt. 29, 516

Vergnole, S., Delage, L., \& Reynaud, F. 2004, Opt. Comm., 232, 31 